\title{
The association between food addiction, disordered eating behaviors and food intake
}

\section{A associação entre dependência alimentar, distúrbios do comportamento alimentar e dependência alimentar}

\section{A B S T R A C T}

\section{Objective}

This study was conducted with 370 undergraduate students to investigate the relationship between food intake, food addiction and disordered eating behavior.

\section{Methods}

The Yale Food Addiction Scale, Eating Attitude Test-26 and Food Frequency Questionnaire were used to assess food addiction, disordered eating behavior and food intake.

\section{Results}

A positive weak correlation was found between the Yale Food Addiction Scale and daily energy, carbohydrate and fat intake $(r=0.228, p<0.001 ; r=0.222, p<0.001 ; r=0.225, p<0.001$ respectively) whereas a negative weak correlation was found between Eating Attitude Test-26 and daily energy and carbohydrate intake $(r=-0.105$, $p=0.044 ; r=-0.116, p=0.025$ respectively).

\section{Conclusion}

Food intake is associated with food addiction and disordered eating behaviour. Further research should be conducted with a larger population also examining body composition, exercises and financial situation of the individuals because all of these factors have an influence on the nutritional status and eating behavior as well.

Keywords: Feeding and eating disorders. Food addiction. Food consumption.

\footnotetext{
1 Eastern Mediterranean University, Faculty of Health Sciences, Department of Nutrition and Dietetics. 99628, Famagusta, North Cyprus via Mersin 10 Turkey. Correspondence to: C GEZER. E-mail: <ceren.gezer@emu.edu.tr>.

Article based from the dissertation of G SENGOR, entitled "Assessment of food addiction, eating disorder and nutritional status of Eastern Mediterranean University students”. Eastern Mediterranean University; 2018.
}

How to cite this article

Sengor G, Gezer C. The association between food addiction, disordered eating behaviors and food intake. Rev Nutr. 2020;33:e190039. http://dx.doi.org/10.1590/1678-9865202033e190039 


\section{RE S U M O}

\section{Objetivo}

Participaram neste estudo 370 estudantes universitários. O objetivo era investigar a relação entre consumo alimentar, dependência alimentar e distúrbios do comportamento alimentar.

\section{Métodos}

A Escala de Dependência Alimentar de Yale, o Teste de Atitudes Alimentares-26 e o Questionário de Frequência Alimentar foram utilizados para avaliar a dependência alimentar, os transtornos do comportamento alimentar e o consumo alimentar.

\section{Resultados}

Verificaram-se uma correlação positiva fraca entre a Escala de Dependência Alimentar de Yale e a ingestão diária de calorias, carboidratos e gorduras $(r=0,228, p<0,001 ; r=0,222, p<0,001 ; r=0,225, p<0,001$ respectivamente) e uma correlação negativa fraca entre o Teste de Atitudes Alimentares-26 e o consumo diário de calorias e carboidratos ( $r=-0,105, p=0,044 ; r=-0,116, p=0,025$ respectivamente).

\section{Conclusão}

O consumo alimentar está associado à dependência alimentar e aos distúrbios do comportamento alimentar. No futuro, devem ser realizadas pesquisas em amostras maiores e devem também ser avaliados a composição corporal, hábitos do exercício físico e a situação financeira dos indivíduos, pois esses fatores influenciam quer a sua situação nutricional, quer os seus comportamentos alimentares.

Palavras-chave: Transtornos da Alimentação e da Ingestão de Alimentos. Dependência de alimentos. Consumo de Alimentos.

\section{NTRODUCTIO N}

The term food addiction involves the idea of certain foods possessing addictive properties, especially foods which are highly processed and energy dense, and overeating of such foods results in an addictive behavior [1]. Food addiction has similarities with substance dependence such as persistence to use/eat, using/eating more than planned etc. Thus the diagnostic scale of food addiction was based on the diagnostic criteria for substance dependence [2,3]. Moreover, disordered eating behaviors and substance dependence have common features. For instance, both binge eating disorder and substance dependence diagnosis include loss of control between food intake and the inability to stop food consumption despite the desire to do so $[4,5]$. This suggests an interconnection between substance dependence, food addiction and disordered eating. Furthermore, it has been suggested that binge eating as a disordered eating behavior might be an addictive behavior due to the addictive properties of the specific foods [6].

The strong motivation behind the compulsive overeating for both food addiction and disordered eating behaviors depends on sweet, salty and fatty foods $[7,8]$. That is why individuals' food consumption can be associated with food addiction and understanding the principles of food addiction is important for the prevention of eating disorders. Restricted eating, vomiting and use of laxatives are some of the unhealthy behaviors which are most commonly seen among university students and those are the factors which can trigger disordered eating behaviors $[9,10]$.

In a study conducted with postgraduate university students, $4.3 \%$ of the students received a food addiction diagnosis [11]. Disordered eating is more prevalent in students aged between 18-25 years, with those individuals also becoming more exposed to the palatable foods in the university environment [12]. 
The studies focusing food intake, food addiction and disordered eating are limited. They mostly focus food addiction and food intake or disordered eating and food intake. In our study we clustered those studies. It is believed that this strategy will make the research more reliable as the three factors are interlinked and affecting each other. In addition, this study is important for assessing the relationship between food intake, food addiction and disordered eating behaviors of university students. Thus, knowing the status and the relationship between food intake, food addiction and disordered eating behaviors of young individuals can help to improve programs and activities not only for preventing eating disorders and food addiction, but also for enhancing healthy eating behaviors. The aim of this study is to investigate the link between food intake, food addiction and disordered eating behaviors of university students.

\section{METHODS}

The study was conducted in the Eastern Mediterranean University, involving 370 undergraduate students. Inclusion criteria of the study involves native Turkish speaking university students with an age range between 18 and 25 years. On the other hand, pregnancy and/or severe psychiatric conditions were exclusion criteria. The sample size was calculated by using a simple random sampling method with a $95 \%$ confidence interval and 5\% sampling error. The frequency rate (rate of having an Eating Attitudes Test-26 [EAT-26] score higher than the critical threshold of 20) was accepted as 0.5 in order to calculate the largest alternative among all possible minimum required sample size possibilities. The participants were randomly selected based on the sample size calculated proportionally to the number of registered faculties students. Subsequently, a questionnaire was used to collect data in face to face interviews by investigators on campus. The study was approved by the Ethical Board of Scientific Research and Publication of the Eastern Mediterranean University numbered 2017/50-36. All participants were asked to sign an informed consent form according to the Declaration of Helsinki.

The questionnaire includes the Yale Food Addiction Scale (YFAS) to assess food addiction, the EAT-26 to assess the disordered eating behaviors and the Food Frequency Questionnaire (FFQ) to evaluate food consumption.

Yale Food Addiction Scale: it was developed and validated by Gearhardt et al. [13]. This scale was then improved accordingly to the DSM-V (Diagnostic and Statistical Munual of Mental Disorders, $5^{\text {th }}$ edition) and was named YFAS 2.0 [14]. Validity and reliability tests in the Turkish version were done by Bayraktar et al. and confidence coefficient was analysed as Cronbach's alpha=0.93 [15]. In this study Chronbach's alpha was calculated to be 0.86 . YFAS includes 25 items and at least 3 points out of 7 and diagnostic criteria of use "causes clinical impairment" should be met for food addiction diagnosis. Increase in the YFAS scores indicates an increased risk of food addiction [14].

Eating Attitude Test-26: was developed and improved by Garner et al. [16]. Validity and reliability of this test in Turkish were performed by Savaşır and Erol and the confidence coefficient was analysed as Cronbach's alpha $=0.70$ [17]. In this study Chronbach's alpha was calculated as 0.87 . The EAT-26 includes 26 items in the form of Likert Scale and the cut off point for EAT-26 is 20 points (out of 78). The increase in the EAT-26 scores reflects increased disordered eating behavior [18].

Food Frequency Questionnaire: it involves amounts and frequency of consumption of 54 food items during the last month. In this study FFQ is adapted for the Turkish population. Consumption 
amounts of each food item were assessed once and then the resulting value was multiplied by the consumption frequency coefficient (each meal: 3.0, everyday: 1.0, 1-2 times in a week: 0.215, 3-4 times in a week: $0.5,5-6$ times in a week: 0.785 , once in 15 days: 0.067 , once a month: 0.033, Never: 0). In order to assess daily consumption amounts [19]. The Nutrition Information Package System (BeBiS 7.2; Ebispro for Windows, Stuttgart, Germany; Turkish Version, 2016). was used to determine the daily dietary intake.

Statistical Analyses: the frequencies, means and standard deviations were found for nominal and ordinal data, respectively. In addition, according to normal distribution analysis results, Mann-Whitney $U$ test or t-test were used to determine statistical significance and Pearson or Spearman Correlation Analysis was used to analyse correlations between YFAS, EAT-26 and macro nutrients. Level of significance was accepted as 0.05 . The analysis of the collected data was performed using SPSS ${ }^{\circledR}$ Statistics 24 (IBM ${ }^{\circledR}$ Corp., New York, 2016).

\section{RE S U L T S}

In Table 1, daily energy and nutrient intake were recorded according to YFAS and gender. Food addicted females exhibit higher energy, fat and carbohydrate intake than non-food addicted females

Table 1. Daily energy and nutrient consumption amounts of individuals according to YFAS and gender. Famagusta, Cyprus, 2018.

\begin{tabular}{|c|c|c|c|c|c|c|c|c|c|c|}
\hline & \multicolumn{5}{|c|}{ Females } & \multicolumn{5}{|c|}{ Males } \\
\hline & \multicolumn{2}{|c|}{ FA $(n=52)$} & \multicolumn{2}{|c|}{ NFA $(n=158)$} & \multirow{2}{*}{$p$} & \multicolumn{2}{|c|}{ FA $(n=26)$} & \multicolumn{2}{|c|}{ NFA $(n=134)$} & \multirow{2}{*}{$p$} \\
\hline & $\bar{x}$ & $( \pm S D)$ & $\bar{x}$ & $( \pm S D)$ & & $\bar{x}$ & $( \pm \mathrm{SD})$ & $\bar{x}$ & $( \pm S D)$ & \\
\hline Energy (kcal) & 2173.0 & $( \pm 879.4)$ & 1883.0 & $( \pm 666.0)$ & $0.013^{*}$ & 2893.8 & $( \pm 654.1)$ & 2640.8 & $( \pm 702.0)$ & 0.913 \\
\hline Protein (g) & 74.4 & $( \pm 27.7)$ & 78.5 & $( \pm 33.4)$ & 0.584 & 108.4 & $( \pm 27.5)$ & 103.4 & $( \pm 35.0)$ & 0.279 \\
\hline Fat (g) & 94.0 & $( \pm 40.5)$ & 84.7 & $( \pm 29.9)$ & $0.029^{*}$ & 121.3 & $( \pm 32.4)$ & 112.7 & $( \pm 34.7)$ & 0.445 \\
\hline Carbohydrate (g) & 247.0 & $( \pm 109.0)$ & 194.5 & $( \pm 86.1)$ & $0.009^{*}$ & 322.4 & $( \pm 92.3)$ & 288.3 & $( \pm 87.0)$ & 0.451 \\
\hline Fibre (g) & 24.6 & $( \pm 8.8)$ & 26.8 & $( \pm 16.2)$ & 0.853 & 33.9 & $( \pm 13.3)$ & 31.3 & $( \pm 13.6)$ & 0.219 \\
\hline Cholesterol (mg) & 300.6 & $( \pm 135.5)$ & 322.4 & $( \pm 139.6)$ & 0.403 & 401.9 & $( \pm 149.1)$ & 395.5 & $( \pm 181.7)$ & 0.390 \\
\hline Vitamin A $(\mu \mathrm{g})$ & 1359.0 & $( \pm 1078.6)$ & 1435.0 & $( \pm 1310.2)$ & 0.801 & 3045.0 & $( \pm 2303.0)$ & 2589.0 & $( \pm 2265.4)$ & 0.222 \\
\hline Vitamin E (mg) & 20.0 & $( \pm 8.7)$ & 20.4 & $( \pm 8.9)$ & 0.853 & 26.1 & $( \pm 9.6)$ & 24.5 & $( \pm 10.0)$ & 0.274 \\
\hline Vitamin B12 $(\mu \mathrm{g})$ & 6.4 & $( \pm 4.5)$ & 6.7 & $( \pm 5.6)$ & 0.778 & 13.0 & $( \pm 9.7)$ & 11.9 & $( \pm 8.9)$ & 0.672 \\
\hline Vitamin B3 (mg) & 14.7 & $( \pm 6.0)$ & 14.8 & $( \pm 5.9)$ & 0.994 & 20.9 & $( \pm 7.5)$ & 20.3 & $( \pm 7.4)$ & 0.670 \\
\hline Vitamin B1 (mg) & 0.9 & $( \pm 0.3)$ & 0.8 & $( \pm 0.3)$ & 0.533 & 1.2 & $( \pm 0.3)$ & 1.1 & $( \pm 0.3)$ & 0.808 \\
\hline Vitamin B2 (mg) & 1.4 & $( \pm 0.5)$ & 1.4 & $( \pm 0.6)$ & 0.954 & 2.1 & $( \pm 0.7)$ & 1.9 & $( \pm 0.7)$ & 0.228 \\
\hline Vitamin B6 (mg) & 1.7 & $( \pm 0.6)$ & 1.6 & $( \pm 0.6)$ & 0.640 & 2.1 & $( \pm 0.6)$ & 2.1 & $( \pm 0.7)$ & 0.566 \\
\hline Folic Acid $(\mu \mathrm{g})$ & 241.7 & $( \pm 83.4)$ & 233.8 & $( \pm 92.3)$ & 0.275 & 350.2 & $( \pm 76.4)$ & 312.4 & $( \pm 88.4)$ & 0.341 \\
\hline Vitamin C (mg) & 49.8 & $( \pm 34.8)$ & 61.3 & $( \pm 39.7)$ & $0.036^{*}$ & 64.0 & $( \pm 39.7)$ & 63.8 & $( \pm 36.8)$ & 0.790 \\
\hline Potassium (mg) & 2532.0 & $( \pm 988.7)$ & 2560.0 & $( \pm 1015.1)$ & 0.835 & 3230.1 & $( \pm 914.9)$ & 3120.8 & $( \pm 988.2)$ & 0.897 \\
\hline Calcium (mg) & 619.4 & $( \pm 252.1)$ & 686.4 & $( \pm 355.3)$ & 0.360 & 837.0 & $( \pm 201.0)$ & 792.4 & $( \pm 292.4)$ & 0.233 \\
\hline Magnesium (mg) & 306.6 & $( \pm 109.8)$ & 296.2 & $( \pm 103.8)$ & 0.334 & 388.2 & $( \pm 109.9)$ & 366.1 & $( \pm 114.6)$ & 0.709 \\
\hline Phosphorus (mg) & 1226.0 & $( \pm 413.8)$ & 1232.0 & $( \pm 435.8)$ & 0.881 & 1659.6 & $( \pm 349.3)$ & 1558.3 & $( \pm 458.4)$ & 0.087 \\
\hline Iron (mg) & 11.9 & $( \pm 4.1)$ & 11.4 & $( \pm 4.3)$ & 0.821 & 16.1 & $( \pm 3.8)$ & 14.9 & $( \pm 4.1)$ & 0.723 \\
\hline Zinc (mg) & 10.3 & $( \pm 3.6)$ & 9.9 & $( \pm 3.4)$ & 0.760 & 14.6 & $( \pm 3.3)$ & 13.6 & $( \pm 3.8)$ & 0.205 \\
\hline
\end{tabular}

Note: *: $p<0.05 ; \bar{X}$ : Mean; FA: Food Addicted; NFA: Non-Food Addicted; SD: Standard Deviation. 
( $p=0.013, p=0.029, p=0.009, p=0.036$ respectively). However, in the case of vitamin $C$ intake, an inverse result was observed. In Table 2, females with higher EAT-26 scores exhibited lower daily energy, protein, carbohydrate, fibre and vitamin C intake $(p<0.05)$.

The Figure 1 shows positive weak correlations between YFAS scores and individuals' daily energy, carbohydrate and fat intake for both genders $(r=0.228, p<0.001 ; r=0.222, p<0.001$; $r=0.225, p<0.001$ respectively). Figure 2 indicates a negative weak correlation between EAT-26 scores and individuals' daily energy and carbohydrate intake $(r=-0.105, p=0.044 ; r=-0.116, p=0.025$ respectively). On the other hand, a positive weak correlation was found between EAT-26 scores and daily fat intake in males $(r=0.185, p=0.019)$.

According to Table 3, daily consumption of cakes, cookies and biscuits were found to be higher in food addicted females in comparison to non-food addicted females $(27.5 \pm 20.1 \mathrm{~g}$ versus $18.1 \pm 18.4 \mathrm{~g})$, while in males an opposite result was found $(20.7 \pm 26.3 \mathrm{~g}$ versus $27.0 \pm 21.3 \mathrm{~g})(p<0.05)$. On the other hand the amount of oily seeds $(71.5 \pm 50.2 \mathrm{~g}$ versus $37.8 \pm 30.6 \mathrm{~g})$, oil and fat consumption $(40.1 \pm 26.2 \mathrm{~g}$ versus $28.5 \pm 16.5 \mathrm{~g})$ was higher in food addicted males than in non-food addicted males $(p<0.05)$. In addition, males with higher disordered eating behavior showed higher consumption of meat and egg than males with lower disordered eating behavior (189.5 $\pm 132.2 \mathrm{~g}$ versus $130.9 \pm 82.0 \mathrm{~g})$ $(p<0.05)$.

Table 2. Daily energy and nutrient intake of individuals according to eating disorder risk and gender. Famagusta, Cyprus, 2018.

\begin{tabular}{|c|c|c|c|c|c|c|c|c|c|c|}
\hline & \multicolumn{5}{|c|}{ Females $(n=210)$} & \multicolumn{5}{|c|}{ Males $(n=160)$} \\
\hline & \multicolumn{2}{|c|}{ EAT- $26 \geq 20$} & \multicolumn{2}{|c|}{ EAT-26 $<20$} & \multirow[b]{2}{*}{$p$} & \multicolumn{2}{|c|}{ EAT- $26 \geq 20$} & \multicolumn{2}{|c|}{ EAT-26 <20 } & \multirow{2}{*}{$p$} \\
\hline & $\bar{x}$ & $( \pm \mathrm{SD})$ & $\bar{x}$ & $( \pm \mathrm{SD})$ & & $\bar{x}$ & $( \pm \mathrm{SD})$ & $\bar{x}$ & $( \pm \mathrm{SD})$ & \\
\hline Energy (kcal) & 1887.0 & $( \pm 823.5)$ & 2003.0 & $( \pm 659.8)$ & $0.045^{*}$ & 2730.0 & $( \pm 659.8)$ & 2663.9 & $( \pm 714.9)$ & 0.299 \\
\hline Protein (g) & 70.9 & $( \pm 30.4)$ & 82.3 & $( \pm 32.5)$ & $0.003^{*}$ & 108.8 & $( \pm 36.0)$ & 102.5 & $( \pm 33.0)$ & 0.652 \\
\hline Fat (g) & 86.5 & $( \pm 37.6)$ & 87.4 & $( \pm 29.3)$ & 0.057 & 120.6 & $( \pm 35.6)$ & 111.6 & $( \pm 33.7)$ & 0.403 \\
\hline Carbohydrate (g) & 199.0 & $( \pm 103.2)$ & 213.8 & $( \pm 88.1)$ & $0.032^{*}$ & 286.6 & $( \pm 77.3)$ & 296.6 & $( \pm 92.5)$ & 0.324 \\
\hline Fibre (g) & 22.4 & $( \pm 13.6)$ & 29.0 & $( \pm 14.9)$ & $<0.001^{*}$ & 31.8 & $( \pm 13.6)$ & 31.7 & $( \pm 13.6)$ & 0.894 \\
\hline Cholesterol (mg) & 297.0 & $( \pm 133.6)$ & 331.6 & $( \pm 140.8)$ & 0.066 & 414.4 & $( \pm 189.8)$ & 389.7 & $( \pm 171.4)$ & 0.627 \\
\hline Vitamin A $(\mu \mathrm{g})$ & 1539.0 & $( \pm 1588.1)$ & 1327.0 & $( \pm 941.8)$ & 0.756 & 2688.0 & $( \pm 2076.1)$ & 2653.7 & $( \pm 2348.6)$ & 0.760 \\
\hline Vitamin E (mg) & 19.0 & $( \pm 8.8)$ & 21.2 & $( \pm 8.7)$ & 0.075 & 26.3 & $( \pm 12.3)$ & 24.1 & $( \pm 8.9)$ & 0.729 \\
\hline Vitamin B12 $(\mu \mathrm{g})$ & 7.1 & $( \pm 6.7)$ & 6.3 & $( \pm 4.1)$ & 0.619 & 12.4 & $( \pm 8.3)$ & 12.0 & $( \pm 9.2)$ & 0.572 \\
\hline Vitamin B3 (mg) & 13.9 & $( \pm 6.2)$ & 15.5 & $( \pm 5.7)$ & 0.516 & 21.2 & $( \pm 7.5)$ & 20.1 & $( \pm 7.3)$ & 0.975 \\
\hline Vitamin B1 (mg) & 0.8 & $( \pm 0.3)$ & 0.9 & $( \pm 0.3)$ & 0.751 & 1.1 & $( \pm 0.3)$ & 1.1 & $( \pm 0.3)$ & 0.156 \\
\hline Vitamin B2 (mg) & 1.4 & $( \pm 0.6)$ & 1.5 & $( \pm 0.6)$ & 0.195 & 2.0 & $( \pm 0.7)$ & 1.9 & $( \pm 0.7)$ & 0.782 \\
\hline Vitamin B6 (mg) & 1.5 & $( \pm 0.6)$ & 1.7 & $( \pm 0.6)$ & 0.517 & 2.1 & $( \pm 0.6)$ & 2.1 & $( \pm 0.7)$ & 0.263 \\
\hline Vitamin C (mg) & 50.0 & $( \pm 39.9)$ & 64.6 & $( \pm 37)$ & $<0.001^{*}$ & 61.0 & $( \pm 33.6)$ & 64.9 & $( \pm 38.5)$ & 0.912 \\
\hline Potassium (mg) & 103.0 & $( \pm 50.0)$ & 116.2 & $( \pm 47.1)$ & 0.981 & 146.1 & $( \pm 49.4)$ & 150.5 & $( \pm 54.6)$ & 0.522 \\
\hline Folic Acid $(\mu \mathrm{g})$ & 2381.0 & $( \pm 1037.9)$ & 2677.0 & $( \pm 968.3)$ & 0.639 & 3180.0 & $( \pm 920.1)$ & 3123.0 & $( \pm 998.1)$ & 0.234 \\
\hline Calcium (mg) & 624.0 & $( \pm 273.1)$ & 703.0 & $( \pm 368.4)$ & 0.081 & 820.3 & $( \pm 287.9)$ & 791.8 & $( \pm 277.2)$ & 0.731 \\
\hline Magnesium (mg) & 278.0 & $( \pm 108.4)$ & 313.6 & $( \pm 100.6)$ & 0.588 & 366.0 & $( \pm 104.5)$ & 371.1 & $( \pm 117.6)$ & 0.172 \\
\hline Phosphorus (mg) & 1152.0 & $( \pm 419.8)$ & 1287.0 & $( \pm 429.1)$ & 0.631 & 1602.0 & $( \pm 449.7)$ & 1564.6 & $( \pm 442.1)$ & 0.725 \\
\hline Iron (mg) & 10.8 & $( \pm 4.5)$ & 12.0 & $( \pm 3.9)$ & 0.211 & 15.4 & $( \pm 3.9)$ & 15.0 & $( \pm 4.1)$ & 0.156 \\
\hline Zinc (mg) & 9.4 & $( \pm 3.5)$ & 10.4 & $( \pm 3.4)$ & 0.311 & 14.4 & $( \pm 3.8)$ & 13.5 & $( \pm 3.7)$ & 0.667 \\
\hline
\end{tabular}

Note: *: $p<0.05 ; \bar{X}$ : Mean; EAT-26: Eating Attitudes Test-26; SD: Standard Deviation. 

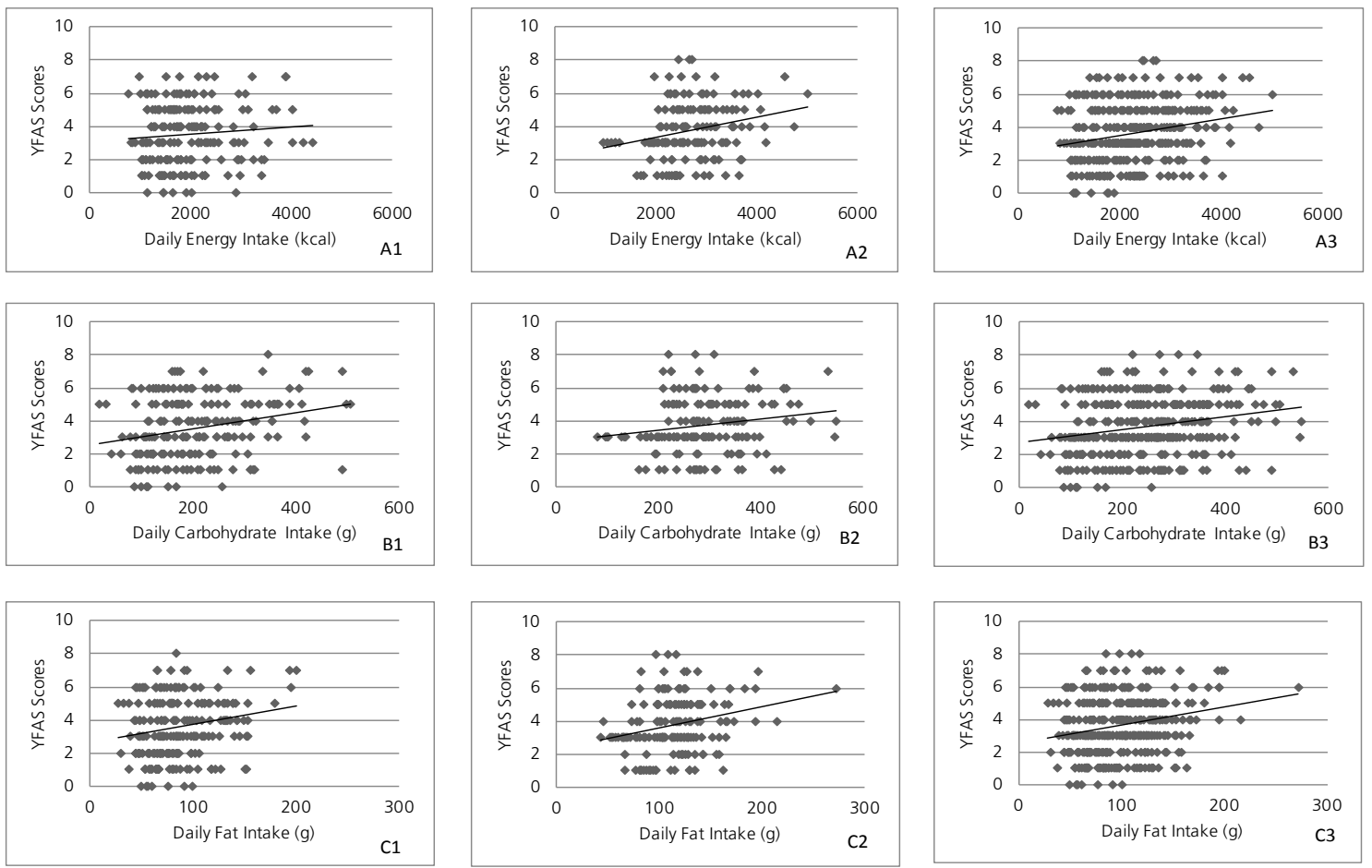

Figure 1. Relationship between YFAS and individual daily energy, carbohydrate and fat intake. Famagusta, Cyprus, 2018. Note: Figures A1-A3: Energy Intake; A1: Female $(r=0.214, p=0.002)$; A2: Male $(r=0.225, p=0.001)$; A3: Total $(r=0.228, p<0.001)$.

Figures B1-B3: Carbohydrate Intake; B1: Female $(r=0.254, p<0.001), B 2$ : Male $(r=0.171, p=0.031)$, B3: Total $(r r=0.222, p<0.001)$.

Figures C1-C3: Fat Intake; C1: Female $(r=0.197, p=0.004)$, C2: Male $(r=0.259, p=0.001), C 3$ : Total $(r=0.225, p<0.001)$.
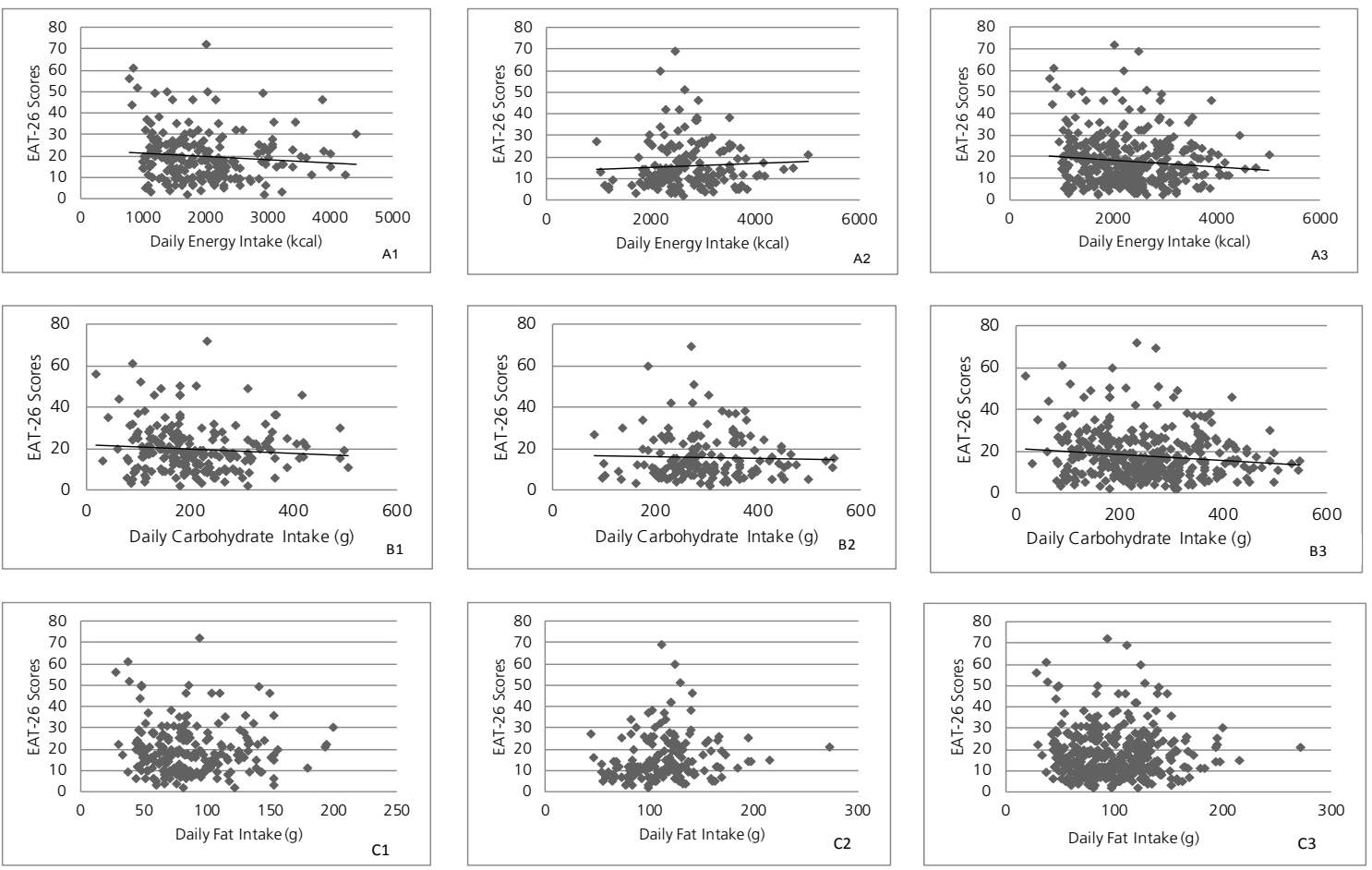

Figure 2. Relationship between EAT-26 and daily energy, carbohydrate and fat intake. Famagusta, Cyprus, 2018.

Note: Figures A1- A3: Energy Intake; A1: Female ( $r=-0.123, p=0.074)$, A2: Male $(r=0.113, p=0.155)$, A3: Total $(r=-0.105, p=0.044)$. Figures B1-B3: Carbohydrate Intake; B1: Female $(r=-0.104, p=0.134)$, B2: Male $(r=0.030, p=0.709), B 3$ : Total $(r=-0.116, p=0.025)$. Figures C1-C3: Fat Intake; C1: Female $(r=-0.034, p=0.620)$, C2: Male $(r=0.185, p=0.019), C 3$ : Total $(r=-0.190, p=0.715)$. 


\section{DISCUSSION}

Food addicted females showed higher energy, carbohydrate and fat intake than non-food addicted females, whereas vitamin $C$ intake was found to be the opposite $(p<0.05)$. In addition, as the daily energy, carbohydrate and fat intake increases YFAS scores increase as well $(r=0.228$, $p<0.001 ; r=0.222 p<0.001 ; r=0.225, p<0.001$ respectively). Similarly, previous studies have found positive correlations between food addiction and daily energy, carbohydrate, fat and protein intake [7,20-22]. However, in another study no relationship between food addiction and carbohydrate, protein and micronutrient intake was found and it was also mentioned that individuals preferred foods which were energy dense and poor in nutrient [23].

The idea of high consumption of these foods is due to their addictive properties and this leads to overconsumption which supports the results of food addicted individuals who eat larger amounts of processed and palatable foods than non-food addicted individuals [24]. In prior studies, food addicted individuals revealed a higher consumption of ready-to-eat food, snacks, french-fries, sugar and soft drinks, with lower consumption of grains, fruit and vegetables than non-food addicted individuals $[23,25]$. Similarly in this study, food addicted females showed a higher consumption of different cakes, cookies and biscuits than non-food addicted females; however, an opposite result was found for males $(p<0.05)$. The results for males was unexpected because the different cakes, cookies and biscuits are high in sugar which have addictive properties and that is why food addicted males were expected to show greater consumption of these foods than non-food addicted males. On the other hand, food addicted males showed a higher consumption of oil, fat and oily seeds as compared to non-food addicted males $(p<0.05)$. Therefore, according to these results, it can be assumed that foods high in fat are more appealing than foods high in sugar for food addicted males, whereas high sugar foods are more attractive for food addicted females.

Moreover, as daily male fat intake increases EAT-26 scores increase as well $(r=0.185, p=0.019)$. Also, males with higher disordered eating behavior exhibited greater consumption of meat and egg than males with lower disordered eating behavior $(189.5 \pm 132.2 \mathrm{~g}$ versus $130.9 \pm 82.0 \mathrm{~g})(p<0.05)$. Thus, it can be deduced that fat increase associated with increased meat and egg intake occurs in males with higher disordered eating behavior. Besides, while individuals' daily energy and carbohydrate intake increases, EAT-26 scores decrease ( $r=-0.105, p=0.044 ; r=-0.116, p=0.025$ respectively). In addition, females with higher disordered eating behavior ingested lower daily energy, protein, carbohydrate, fibre and vitamin $C$ than females with lower disordered eating behavior $(p<0.05)$.

In a study conducted with female swimmers, those with high disordered eating behavior ingested lower energy, carbohydrate, fat, iron and zinc, and higher vitamin $C$ and folate intake than females with low disordered eating behavior [26]. Another study showed that in females, as the disordered eating behavior increases, inadequate intake of energy and nutrients also increases, while in males only vitamin and mineral intake decreases with disordered eating behaviour [27]. Thus, unhealthy eating behavior among females could be due to hormonal, social and psychological factors.

\section{CONCLUSION}

Food addiction was positively correlated with daily energy, carbohydrate and fat intake, while a negative correlation was found between the disordered eating behavior and daily energy and carbohydrate intake. Therefore, food intake is associated with both food addiction and disordered 
Table 3. Daily consumption of food groups according to eating disorder risk, food addiction and gender (g/day). Famagusta, Cyprus, 2018.

\begin{tabular}{|c|c|c|c|c|c|c|c|c|c|c|}
\hline & \multicolumn{5}{|c|}{ Females $(n=210)$} & \multicolumn{5}{|c|}{ Males $(n=160)$} \\
\hline & \multicolumn{2}{|c|}{ FA } & \multicolumn{2}{|c|}{ NFA } & \multirow{2}{*}{$p$} & \multicolumn{2}{|r|}{ FA } & \multicolumn{2}{|c|}{ NFA } & \multirow[b]{2}{*}{ p } \\
\hline & $\bar{x}$ & $( \pm S D)$ & $\bar{x}$ & $( \pm S D)$ & & $\bar{x}$ & $( \pm S D)$ & $\bar{x}$ & $( \pm S D)$ & \\
\hline $\begin{array}{l}\text { Milk and Dairy } \\
\text { Products }\end{array}$ & 270.8 & $( \pm 169.6)$ & 226.3 & $( \pm 111.5)$ & 0.122 & 243.5 & $( \pm 116.4)$ & 304.6 & $( \pm 207.0)$ & 0.259 \\
\hline $\begin{array}{l}\text { Group of Bread } \\
\text { and Grains }\end{array}$ & 306.0 & $( \pm 128.5)$ & 296.7 & $( \pm 130.2)$ & 0.924 & 329.0 & $( \pm 147.5)$ & 295.1 & $( \pm 135.2)$ & 0.565 \\
\hline Oily Seeds & 42.8 & $( \pm 25.0)$ & 42.2 & $( \pm 32.7)$ & 0.565 & 71.5 & $( \pm 50.2)$ & 37.8 & $( \pm 30.6)$ & $<0.001^{*}$ \\
\hline $\begin{array}{l}\text { Group of Meat } \\
\text { and Egg }\end{array}$ & 125.1 & $( \pm 58.9)$ & 102.7 & $( \pm 70.6)$ & $0.002^{*}$ & 154.6 & $( \pm 89.2)$ & 175.6 & $( \pm 110.4)$ & 0.508 \\
\hline Sausage & 8.9 & $( \pm 7.4)$ & 8.2 & $( \pm 8.2)$ & $0.018^{*}$ & 11.0 & $( \pm 10.9)$ & 8.8 & $( \pm 8.9)$ & 0.332 \\
\hline Vegetables & 96.5 & $( \pm 71.1)$ & 87.6 & $( \pm 92.4)$ & 0.052 & 115.2 & $( \pm 110.7)$ & 162.6 & $( \pm 137.2)$ & 0.919 \\
\hline $\begin{array}{l}\text { Potato, Starch and } \\
\text { Mushroom }\end{array}$ & 118.1 & $( \pm 59.2)$ & 105.0 & $( \pm 71.1)$ & 0.080 & 140.0 & $( \pm 47.9)$ & 127.1 & $( \pm 68.2)$ & 0.279 \\
\hline Fruits & 83.7 & $( \pm 66.2)$ & 85.1 & $( \pm 69.6)$ & 0.974 & 95.5 & $( \pm 71.2)$ & 134.8 & $( \pm 129.2)$ & 0.207 \\
\hline Sweets & 66.3 & $( \pm 43.0)$ & 42.5 & $( \pm 37.8)$ & 0.054 & 53.0 & $( \pm 46.9)$ & 78.0 & $( \pm 61.0)$ & 0.066 \\
\hline $\begin{array}{l}\text { Cakes, Cookies } \\
\text { and Biscuits }\end{array}$ & 27.5 & $( \pm 20.1)$ & 18.1 & $( \pm 18.4)$ & $0.001^{*}$ & 20.7 & $( \pm 26.3)$ & 27.0 & $( \pm 21.3)$ & $0.034^{*}$ \\
\hline \multirow[t]{3}{*}{ Oil and Fat } & 31.1 & $( \pm 14.0)$ & 30.8 & $( \pm 16.3)$ & 0.773 & 40.1 & $( \pm 26.2)$ & 28.5 & $( \pm 16.5)$ & $0.041^{*}$ \\
\hline & \multicolumn{2}{|c|}{ EAT- $26 \geq 20$} & \multicolumn{2}{|c|}{ EAT-26 $<20$} & & \multicolumn{2}{|c|}{ EAT- $26 \geq 20$} & \multicolumn{2}{|c|}{ EAT-26 $<20$} & \\
\hline & $\bar{x}$ & $( \pm S D)$ & $\bar{x}$ & $( \pm S D)$ & & $\bar{x}$ & $( \pm S D)$ & $\bar{x}$ & $( \pm S D)$ & $\rho$ \\
\hline $\begin{array}{l}\text { Milk and Dairy } \\
\text { Products }\end{array}$ & 247.3 & $( \pm 137.8)$ & 230.1 & $( \pm 122.9)$ & 0.476 & 272.3 & $( \pm 150.0)$ & 303.1 & $( \pm 211.0)$ & 0.412 \\
\hline $\begin{array}{l}\text { Group of Bread } \\
\text { and Grains }\end{array}$ & 295.6 & $( \pm 124.9)$ & 301.5 & $( \pm 133.2)$ & 0.292 & 275.0 & $( \pm 121.9)$ & 310.3 & $( \pm 142.1)$ & 0.196 \\
\hline Oily Seeds & 44.6 & $( \pm 27.8)$ & 40.8 & $( \pm 33.1)$ & 0.168 & 40.5 & $( \pm 40.3)$ & 44.3 & $( \pm 35.1)$ & 0.370 \\
\hline $\begin{array}{l}\text { Group of Meat } \\
\text { and Egg }\end{array}$ & 167.5 & $( \pm 98.8)$ & 98.5 & $( \pm 58.7)$ & $<0.001^{*}$ & 189.5 & $( \pm 132.2)$ & 130.9 & $( \pm 82.0)$ & $0.003^{*}$ \\
\hline Sausage & 8.3 & $( \pm 7.6)$ & 8.4 & $( \pm 8.3)$ & 0.083 & 7.8 & $( \pm 7.9)$ & 9.7 & $( \pm 9.7)$ & 0.388 \\
\hline Vegetables & 102.1 & $( \pm 94.1)$ & 80.9 & $( \pm 81.7)$ & 0.084 & 135.1 & $( \pm 108.5)$ & 171.3 & $( \pm 140.3)$ & 0.124 \\
\hline Fruits & 88.0 & $( \pm 78.0)$ & 82.4 & $( \pm 61.3)$ & 0.993 & 123.6 & $( \pm 126.2)$ & 130.2 & $( \pm 121.4)$ & 0.443 \\
\hline Sweets & 50.3 & $( \pm 42.6)$ & 47.0 & $( \pm 38.9)$ & 0.199 & 90.4 & $( \pm 70.9)$ & 67.7 & $( \pm 53.6)$ & $0.015^{*}$ \\
\hline $\begin{array}{l}\text { Cakes, Cookies } \\
\text { and Biscuits }\end{array}$ & 27.0 & $( \pm 22.4)$ & 20.5 & $( \pm 19.0)$ & 0.054 & 26.3 & $( \pm 18.4)$ & 20.9 & $( \pm 21.6)$ & 0.289 \\
\hline Oil and Fat & 30.9 & $( \pm 13.6)$ & 30.9 & $( \pm 17.1)$ & 0.876 & 27.8 & $( \pm 19.7)$ & 31.3 & $( \pm 18.5)$ & 0.174 \\
\hline
\end{tabular}

Note: *: $p<0.05 ; \bar{X}$ : Mean; FA: Food Addicted; NFA: Non-Food Addicted; SD: Standard Deviation.

eating behavior but is inversely related. This study's results are important because both food addiction and disordered eating behavior relationship with food intake have been shown in one sample. However, further research should be conducted with a larger population, also examining body composition, exercises and financial situation of the individuals because all these factors have an influence on the nutritional status and eating behaviors of the individuals.

\section{CONTRIBUTORS}

G SENGOR and C GEZER had equivalent roles in the study conception and design, data analysis and interpretation. 


\section{REFERE NCES}

1. Özkan I, Devrim A, Bilgiç P. Evaluation of the relationship between food addiction, nutritional status and depression in overweight and obese women. J Nutr Diet. 2017;45(3):242-9.

2. Long CG, Blundell JE, Finlayson G. A systematic review of the application and correlates of YFAS-diagnosed food addiction in humans: are eating-related addictions a cause for concern or empty concepts?. Obes Facts. 2015;8(6):386-401.

3. Penzenstadler L, Soares C, Karila L, Khazaal Y. Systematic review of food addiction as measured with the Yale Food Addiction Scale: implications for the food addiction construct. Curr Neuropharmacol. 2018;16:1-13.

4. Davis C. A commentary on the associations among food addiction, binge eating disorder, and obesity: overlapping conditions with idiosyncratic clinical features. Appetite. 2017;115:3-8.

5. Treasure J, Leslie M, Chami R, Fernandez-Aranda F. Are trans diagnostic models of eating disorders fit for purpose? A consideration of the evidence for food addiction. Eur Eat Disorders Rev. 2017;26:83-91.

6. Meule A, Gearhardt AN. Food addiction in the light of DSM-5. Nutrients. 2014;6(9): 3653-71.

7. Dimitrijević I, Popović N, Sabljak V, Škodrić-Trifunović V, Dimitrijević N. Food addiction-diagnosis and treatment. Psychiatr Danub. 2015;27(1):101-6.

8. Schulte EM, Avena NM, Gearhardt AN. Which foods may be addictive? The roles of processing, fat content, and glycemic load. Plos One. 2015;10(2):e0117959. http://dx.doi.org/10.1371/journal.pone.0117959

9. Yu Z, Tan M. Disordered eating behaviors and food addiction among nutrition major college students. Nutrients. 2016;8(11):E673. http://dx. doi.org/10.3390/nu8110673

10. Pengpid S, Peltzer K, Ahsan GU. Risk of eating disorders among university students in Bangladesh. Int J Adolesc Med Health. 2015;27(1):93-100.

11. Manzoni GM, Rossi A, Pietrabissa G, Varallo G, Molinari E, Poggiogalle E, et al. Validation of Italian Yale Food Addiction Scale in postgraduate university students. Eat Weight Disord. 2018;23:167-76.

12. Yu Z, Indelicato NA, Fuglestad P, Tan M, Lindsay B, Sticea C. Sex differences in disordered eating and food addiction among college students. Appetite. 2018;129:12-8. http://dx.doi.org/10.1016/j.appet.2018.06. 028

13. Gearhardt AN, Corbin WR, Brownell KD. Preliminary validation of the Yale Food Addiction Scale. Appetite. 2009;52(2):430-6.

14. Gearhardt AN, Corbin WR, Brownell KD. Development of the Yale Food Addiction Scale Version 2.0. Psychol Addict Behav. 2016;30(1):113-21.

15. Bayraktar F, Erkman F, Kurtuluş E. Adaptation study of Yale Food Addiction Scale. Klin Psikofarmakol B. 2012;22:38.

16. Garner DM, Olmsted MP, Bohr Y, Garfinkel PE. The eating attitudes test: psychometric features and clinical correlates. Psychol Med. 1982;12(4):871-8.

17. Savaşır I, Erol N. Yeme tutum testi: anoreksiya nervoza belirtileri indeksi. Turk J Psychol. 1989;7(23):19-25.

18. Rodríguez MA, Salar VN, Carretero MC, Gimeno CE, Collado RE. Eating disorders and diet management in contact sports: EAT-26 questionnaire does not seem appropriate to evaluate eating disorders in sports. Nutr Hosp. 2015;32(4):1708-14.

19. Pekcan G. Beslenme durumunun saptanması. In: Baysal A, et al. Diyet El Kitabı. Ankara: Hatiboğlu Yayınları; 2016.

20. Lemeshow AR, Rimm EB, Hasin DS, Gearhardt AN, Flint AJ, Field AE, et al. Food and beverage consumption and food addiction among women in the Nurses' Health Studies. Appetite. 2018;121:186-97.

21. Küçükerdönmez Ö, Urhan M, Altın M, Hacıraifoğlu O, Yıldız B. Assessment of the relationship between food addiction and nutritional status in schizophrenic patients. Nutr Neurosci. 2017;1-9.

22. Ayaz A, Nergiz-Unal R, Dedebayraktar D, Akyol A, Pekcan AG, Besler HT, et al. How does food addiction influence dietary intake profile? Plos One. 2018;13(4):e0195541. http://dx.doi.org/10.1371/journal.pone.019 5541 
23. Pursey KM, Collins CE, Stanwell P, Burrows TL. Foods and dietary profiles associated with food addiction in young adults. Addict Behav Rep. 2015;5(2):41-8.

24. Carlier N, Marshe VS, Cmorejova J, Davis C, Müller DJ. Genetic similarities between compulsive overeating and addiction phenotypes: a case for food addiction?. Curr Psychiatry Rep. 2015;17(12):96.

25. Burrows T, Hides L, Brown R, Dayas CV, Kay-Lambkin F. Differences in dietary preferences, personality and mental health in australian adults with and without food addiction. Nutrients. 2017;9(3):285.

26. Costa NF, Schtscherbyna A, Soares EA, Ribeiro BG. Disordered eating among adolescent female swimmers: dietary, biochemical, and body composition factors. Nutrition. 2013; 29(1):172-7.

27. Aparicio-Llopis E, Canals J, Arija V. Dietary intake according to the course of symptoms of eating disorders in a school-based follow-up study of adolescents. Eur Eat Disord Rev. 2014;22(6):412-42.

Received: February 28, 2019

Final Version: November 19, 2019

Approved: December 3, 2019 